\title{
MEDICAL, SCENE AND EVENT IMAGE CATEGORY RECOGNITION USING COMPLETED LOCAL TERNARY PATTERNS (CLTP)
}

\author{
Taha H. Rassem ${ }^{1}$, Bee Ee Khoo², Mohammed Falah Mohammed ${ }^{3}$, Nasrin M.Makbol ${ }^{4}$
}

${ }^{1,3}$ Faculty of Computer Systems and Software Engineering

${ }^{1}$ IBM Centre of Excellence

Universiti Malaysia Pahang,

26300, Kuantan, Malaysia

Tel: 609-5492245

Fax:609-5492214

${ }^{2,4}$ School of Electrical and Electronic Engineering

Universiti Sains Malaysia, Engineering Campus

14300 Nibong Tebal, Seberang Perai Selatan, Pulau Pinang, Malaysia

Tel : 045941022

Fax : 045941023

Email: tahahussein@ump.edu.my ${ }^{1}$, beekhoo@usm.my ${ }^{2}$,falah@ump.edu.my ${ }^{3}$,nasrin_makbol@usm.my ${ }^{4}$

\begin{abstract}
Many of texture descriptors are proposed based on the Local Binary Pattern (LBP) and have been achieved remarkable texture classification accuracy such as Completed LBP $(C L B P)$ and Completed Local Binary Count (CLBC). However, the LBP suffers from two weaknesses where: 1) it is sensitive to noise and;2) it sometimes classify two or more different patterns falsely to the same class. To overcome the LBP weaknesses, we propose a new texture descriptor which is defined as Completed Local Ternary Pattern (CLTP). The CLTP was used for rotation invariant texture classification. It demonstrates superior texture classification accuracy as compared to CLBP and CLBC descriptors. This is because, the CLTP is more robust to noise and has a high discriminating property that achieves impressive classification accuracy rates. In this paper, two types of experiments are carried out. In the first experiment, different amount of additive Gaussian noise is added to the TC10 Outex texture data set to investigate and prove the robustness of the CLTP against the noise. For the second experiment, the performance of CLTP for image category recognition is studied and investigated. A variety of image datasets are used in the experiments such as scene data set (e.g., Oliva and Torralba datasets (OT8)), Event sport datasets, 2D HeLa medical images, and our new scene data set, defined as USM scene data set. The experimental results proved the superiority of the CLTP descriptor over the original LBP, and different new texture descriptors such as CLBP in the image category recognition, as well as the robustness against the noise. In $2 D$ HeLa medical images, the proposed CLTP has achieved the highest state of the art classification rate reaching $95.62 \%$.
\end{abstract}

Keywords: Completed Local Ternary Pattern descriptor (CLTP), Texture Classification, Image Category Recognition, 2D HeLa images.

\subsection{INTRODUCTION}

Recently, texture descriptors become one of the important descriptors in many applications such as image retrieval [1], image categorisation [2], face recognition [3], and human detector [4]. There are a few numbers of texture descriptors proposed and investigated for different kind of applications. Many of these descriptors are briefly reviewed in many review papers [5]-[7]. Zhang et al. [5] classified the texture feature algorithm methods 
into three different categories which are statistical algorithm methods, model-based methods, and structural methods.

One of the famous texture descriptors is Local Binary Pattern (LBP) which proposed by Ojala et al. [8]. To represent the image texture, they calculated the absolute difference between the gray level of the center pixel of a specific local pattern and its neighbors to construct a texton histogram. Later, they improved their work and used the sign of the differences between the gray level of the center pixel and its neighbors of the local pattern instead of magnitude, in order to propose the LBP texture descriptor [9]. The LBP is shown in Fig. 1. Due to the ability of LBP to distinguish the micro-structures of an image such as edges, lines and spots, it becomes one of the interesting research for many computer vision researchers. Although, the LBP is proposed for rotation invariant texture classification, also it has been used for many applications such as face recognition [3], image retrieval [1], human detector [4], and image categorisation [2]. Many of the variants of the LBP have been suggested and proposed for rotation invariant texture classification such as the Center-symmetric Local Binary Pattern (CS- LBP) [10], Dominant LBP (DLBP) [11], Local Ternary Pattern (LTP) [12], completed LBP ( CLBP) [13], DNS-LBP [14], Local Binary Count (CLBC) [15], Completed Local Ternary Pattern (CLTP) [16], and Local Orientation Adaptive Descriptor [24].

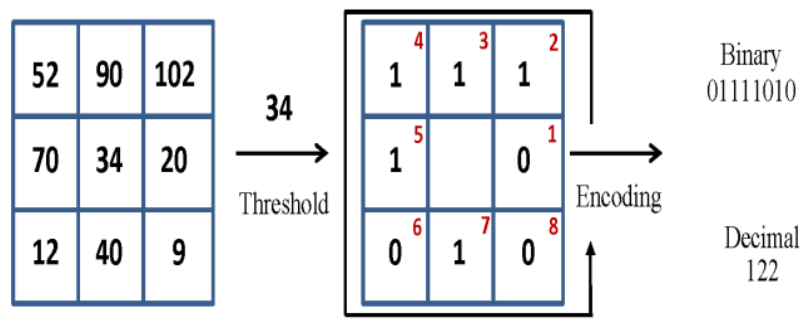

Fig. 1: LBP operator

To overcome the limitations of LBP where it is sensitive to noise and sometimes may classify two or more different patterns falsely to the same class, Raseem and Khoo [16] proposed CLTP . Although, the LTP descriptor is more robust to noise than LBP, however, the latter weakness may appear with the LTP as well as with LBP. The experimental results illustrated that CLTP is more robust to noise, rotation and illumination variance, and it achieves a higher texture classification rates compare to CLBP and CLBC. The proposed CLTP descriptors are evaluated using different types of texture datasets only [16]. Due to the importance of the texture descriptors in many applications, the proposed CLTP should be able to perform well for image classification such as scene, event, and medical image categorization.

This paper presents two different experiments which have been conducted to evaluate the CLTP. The first experiment was conducted on the Outex texture database (TC10) to demonstrate the resistance of the CLTP against the noise. In this experiment, the testing image was corrupted by Additive Gaussian Noise with zero mean and standard deviation that was determined according to the corresponding Signal-to-Noise Ratios (SNR) value. For the second experiment, we studied and evaluated the performance of CLTP for image categorisation. The performances of the proposed CLTP descriptors are evaluated and analysed experimentally for image category recognition using different types of image datasets. These datasets are scene image dataset such as Oliva and Torralba dataset (OT8) [17], Event sport datasets [18], medical image dataset such as 2D HeLa images [19], and our new scene dataset, known as USM scene dataset. The experimental results show that CLTP is more robust to noise, and it achieves better classification accuracy rates than CLBP for image category recognition as well as for texture classification as proved in [16].

The rest of this paper is organised as follows. In Section 2, LBP and CLBP are briefly reviewed, respectively. Our proposed CLTP texture descriptors are explained in Section 3. Then in Section 4, the experimental results of robustness of CLTP against the noise and the experimental results of the OT8, the Event sport, 2D HeLa, USM image datasets are reported and discussed. Finally, Section 5 concludes the paper. 


\subsection{RELATED WORK}

\subsection{Local Binary Pattern (LBP)}

The LBP calculation can be mathematically described as,

$$
L_{B, R}=\sum_{p=0}^{P-1} 2^{p} s\left(i_{p}-i_{c}\right), \quad s(x)= \begin{cases}1, & x>=0 \\ 0, & x<0\end{cases}
$$

(1)

where $i_{c}$ and $i_{p}(\mathrm{p}=0, \ldots, \mathrm{P}-1)$ denote the gray values of the center pixel and the neighbor pixel on a circle of radius $\mathrm{R}$, respectively, and $\mathrm{P}$ denotes the number of the neighbors. To estimate the neighbors that do not lie exactly in the center of the pixels, the bilinear interpolation estimation method is used.

\begin{tabular}{|l|l|l|}
\hline 46 & 46 & 45 \\
\hline 51 & 52 & 52 \\
\hline 50 & 52 & 53 \\
\hline
\end{tabular}

LBP 100000011

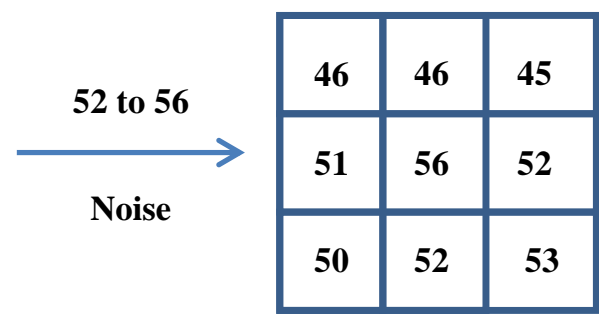

LBP 00000000

Fig.2: The example for LBP operator's noise sensitivity

\begin{tabular}{|c|c|c|}
\hline 52 & 90 & 102 \\
\hline 70 & 34 & 20 \\
\hline 12 & 40 & 9 \\
\hline
\end{tabular}

\begin{tabular}{|c|c|c|}
\hline 130 & 200 & 95 \\
\hline 92 & 90 & 70 \\
\hline 12 & 100 & 9 \\
\hline
\end{tabular}

\begin{tabular}{|l|l|l|}
\hline 1 & 1 & 1 \\
\hline 1 & & 0 \\
\hline 0 & 1 & 0 \\
\hline
\end{tabular}

\begin{tabular}{|l|l|l|}
\hline 1 & 1 & 1 \\
\hline 1 & & 0 \\
\hline 0 & 1 & 0 \\
\hline
\end{tabular}

\section{LBP 01111010}

Fig.3: Similar LBP codes for two different texture patterns.

In addition to LBP, Ojala et al. [9] also improved the original LBP to rotation invariant LBP ( $\left.L B P_{P, R}^{r i}\right)$ and uniform rotation invariant LBP ( $L B P_{P, R}^{r i u 2}$ ). After doing the encoding step in any of these LBP types; i.e., LBP, $L B P_{P, R}^{r i}$ and $L B P_{P, R}^{r i u 2}$, the descriptor histogram is constructed according to Equation (2) as follows: 


$$
H(k)=\sum_{i=0}^{I} \sum_{j=0}^{J} f\left(L B P_{P, R}(i, j), k\right), \quad k \in[0, K], \quad f(x, y)=\left\{\begin{array}{lc}
1, & x=y, \\
0, & \text { otherwise }
\end{array}\right.
$$

where $\mathrm{K}$ is the maximal LBP pattern value.

Since 2002, many researchers have targeted the LBP duo to its characteristics [12,13,15]. One of newest texture feature inspired from the LBP descriptor is the Completed Modeling of Local Binary Pattern (CLBP) [13]. The CLBP show an impressive performance for texture classification task. Texture patterns can be one of the important components of images, especially for the natural scene images. Nonetheless, the LBP still suffers from the sensitivity to noise, and different patterns of LBP may be wrongly classified into the same class that reduces its discriminating property. These weaknesses can be shown in Fig. 2 and Fig. 3, respectively.

The CLBP inherited these flaws. Therefore, it is important to investigate how these flaws can be addressed to improve and enhance their performace.

\subsection{Completed Local Binary Pattern (CLBP)}

In 2010, Guo et al. [12] proposed the completed LBP (CLBP) descriptor. In CLBP, the image local difference is decomposed into two complementary components; the sign component $s_{p}$ and the magnitude component $m_{p}$.

$$
\left.s_{p}=s\left(i_{p}-i_{c}\right), \quad m_{p}=\mid i_{p}-i_{c}\right) \mid
$$

Then, the $s_{p}$ is used to build the CLBP-Sign (CLBP_S), whereas the $m_{p}$ is used to build CLBP-magnitude $\left(C L B P \_M\right)$. The $C L B P \_S$ and $C L B P \_M$ are mathematically described as follows:

$$
\begin{gathered}
C L B P_{-} S_{P, R}=\sum_{p=0}^{P-1} 2^{p} s\left(i_{p}-i_{c}\right) \quad s_{P}= \begin{cases}1, & i_{p} \geq i_{c}, \\
0, & \text { otherwise },\end{cases} \\
C L B P_{-} M_{P, R}=\sum_{p=0}^{P-1} 2^{p} t\left(m_{p}, c\right) \quad t\left(m_{p}, c\right)= \begin{cases}1, & \left.\mid i_{p}-i_{c}\right) \mid \geq c, \\
0, & \left.\mid i_{p}-i_{c}\right) \mid<c,\end{cases}
\end{gathered}
$$

where $i_{c}, i_{p}, \mathrm{R}$ and $\mathrm{P}$ are defined before in Equation (1), while $c$ denotes the mean value of $m_{p}$ in the whole image.

The $C L B P \_S$ is equal to LBP whereas the $C L B P_{-} M$ measures the local variance of magnitude. Furthermore, Guo et al. [12] used the value of the grey level of each pattern to construct a new operator, called CLBP-centre (CLBP_C). The CLBP_C can be mathematically described as follows:

$$
C L B P_{-} C_{P, R}=t\left(i_{c}, c_{I}\right)
$$

where $i_{c}$ denotes the grey value of the centre pixel and $c_{I}$ is the average grey level of the whole image.

Guo et al. [12] combined their operators into joint or hybrid distributions and achieved remarkable texture classification accuracy. They combined $C L B P_{-} S$ and $C L B P_{-} M$ in two ways. In the first way, they concatenated their histogram to build $C L B P_{-} S_{-} M$, while in the second way, they calculated the 2D joint histogram. This 2D joint histogram is known as $C L B P \_S / M$. CLBP_C is also combined with $C L B P \_S$ and $C L B P \_M$ in two ways. In the first way, both of them are combined as 3D joint histogram and denoted as $C L B P \_S / M / C$. In the second way, the $C L B P_{-} C$ is first combined jointly with the $C L B P_{-} S$ or $C L B P_{-} M$ to build $2 \mathrm{D}$ joint histogram denoted $C L B P_{-} S / C$ or $C L B P_{-} M / C$, respectively. Then, this $2 \mathrm{D}$ joint histogram has to be converted to $1 \mathrm{D}$ histogram and 
has to be concatenated with $C L B P \_M$ or $C L B P \_S$ to build the final histogram that denoted by $C L B P \_M \_S / C$ or $C L B P \_S \_M / C$.

\subsection{COMPLETED LOCAL TERNARY PATTERN (CLTP)}

In CLTP[16], local difference of the image is decomposed into two sign complementary components and two magnitude complementary components as follows:

$$
\begin{array}{ll}
s_{p}^{\text {upper }}=s\left(i_{p}-\left(i_{c}+t\right)\right), & s_{p}^{\text {lower }}=s\left(i_{p}-\left(i_{c}-t\right)\right) \\
m_{p}^{\text {upper }}=\left|i_{p}-\left(i_{c}+t\right)\right|, & m_{p}^{\text {lower }}=\left|i_{p}-\left(i_{c}-t\right)\right|
\end{array}
$$

where $i_{c}$, and $i_{p}$ are defined as before in (1) while $t$ denotes the user Threshold.

The $s_{p}^{\text {upper }}$ and $s_{p}^{\text {lower }}$ are used to build the $C L T P_{-} S_{P, R}^{\text {upper }}$ and $C L T P P_{-} S_{P, R}^{\text {lower }}$, respectively, as follows:

$$
\begin{gathered}
C L T P_{-} S_{P, R}^{\text {upper }}=\sum_{p=0}^{P-1} 2^{p} s\left(i_{p}-\left(i_{c}+t\right)\right) \quad s_{P}^{\text {upper }}= \begin{cases}1, & i_{p}>i_{c}+t \\
0, & \text { otherwise },\end{cases} \\
C L T P_{-} S_{P, R}^{\text {lower }}=\sum_{p=0}^{P-1} 2^{p} s\left(i_{p}-\left(i_{c}-t\right)\right) \quad s_{P}^{\text {lower }}= \begin{cases}1, & i_{p}>i_{c}-t, \\
0, & \text { otherwise },\end{cases}
\end{gathered}
$$

Then $C L T P{ }_{-} S_{P, R}$ is the concatenation of the $C_{2 T P} S_{P, R}^{\text {upper }}$ and $C L T P{ }_{-} S_{P, R}^{\text {lower }}$, as follows:

$$
C L T P_{-} S_{P, R}=\left[C L T P_{-} S_{P, R}^{\text {upper }} \quad C L T P_{-} S_{P, R}^{\text {lower }}\right]
$$

Similar to $C L T P_{-} S_{P, R}$, the $C_{2 T P} M_{P, R}$ is built using the two magnitude complementary components $m_{p}^{\text {upper }}$ and $m_{p}^{\text {lower }}$, as follows:

$$
C L T P_{-} M_{P, R}^{\text {upperr }}=\sum_{p=0}^{P-1} 2^{p} t\left(m_{p}^{\text {upper }}, c\right) \quad t\left(m_{p}^{\text {upper }}, c\right)= \begin{cases}1, & \left|i_{p}-\left(i_{c}+t\right)\right|>=c, \\ 0, & \left|i_{p}-\left(i_{c}+t\right)\right|<c\end{cases}
$$




$$
\begin{aligned}
& C L T P_{-} M_{P, R}^{\text {lower }}=\sum_{p=0}^{P-1} 2^{p} t\left(m_{p}^{\text {lower }}, c\right) \quad t\left(m_{p}^{\text {lower }}, c\right)= \begin{cases}1, & \left|i_{p}-\left(i_{c}-t\right)\right|>=c, \\
0, & \left|i_{p}-\left(i_{c}-t\right)\right|<c\end{cases} \\
& C L T P \_M_{P, R}=\left[C L T P \_M_{P, R}^{\text {upper }} \quad C L T P{ }_{-} M_{P, R}^{\text {lower }}\right]
\end{aligned}
$$

Moreover, the $C L T P \_C_{P, R}^{\text {upper }}$ and $C L T P \_C_{P, R}^{\text {lower }}$ can be described mathematically as follows:

$$
\begin{aligned}
& C L T P \_C_{P, R}^{\text {upper }}=t\left(i_{c}^{\text {upper }}, c_{I}\right) \\
& C L T P \_C_{P, R}^{\text {loerr }}=t\left(i_{c}^{\text {lower }}, c_{I}\right)
\end{aligned}
$$

where $i_{c}^{\text {upper }}=i_{c}+t, i_{c}^{\text {lower }}=i_{c}-t$ and $c_{I}$ is the average gray level of the world image.

The extraction process of CLTP can be summarized in Figure 4. The proposed CLTP operators are combined into joint or hybrid distributions to build the final operator histogram like the CLBP and CLBC [13], [15]. In the CLTP, the operators of the same type of pattern; i.e., the upper and the lower pattern, are first combined into joint or hybrid distributions. Then, their results are concatenated to build the final operator histogram.

\subsection{EXPERIMENTS AND DISCUSSIONS}

To evaluate the CLTP texture descriptor, two kinds of experiments are performed. The first experiment was conducted on the Outex texture database (TC10) to demonstrate the effectiveness of the CLTP against the noise. In the second experiment, the OT8, Event sport, 2D HeLa medical image, and our new scene data set, known as USM scene data set are used to investigate and evaluate the performance of the proposed CLTP for image category recognition. In both experiments, the CLTP performances are compared with CLBP descriptor.

Empirically, the threshold value $\mathrm{t}$ is set to 5 in all CLTP experiments. Different datasets were used in order to find the suitable threshold value which will be used in the CLTP evaluation experiments. The values were ranged from 0 to 25 , and 5 was the suitable threshold value.

In these experiments, chi-square statistic is used to measure the dissimilarity between two histograms while the nearest neighborhood classifier is used for classification. The $\chi^{2}$ distance between two histogram $H=h_{i}$ and $K=k_{i}$ where $(i=1,2,3, \ldots B)$ can be mathematically described as follows:

$$
\text { Dissimilarity }_{\chi^{2}}(H, K)=\sum_{i=1}^{B} \frac{\left(h_{i}-k_{i}\right)^{2}}{h_{i}+k_{i}}
$$




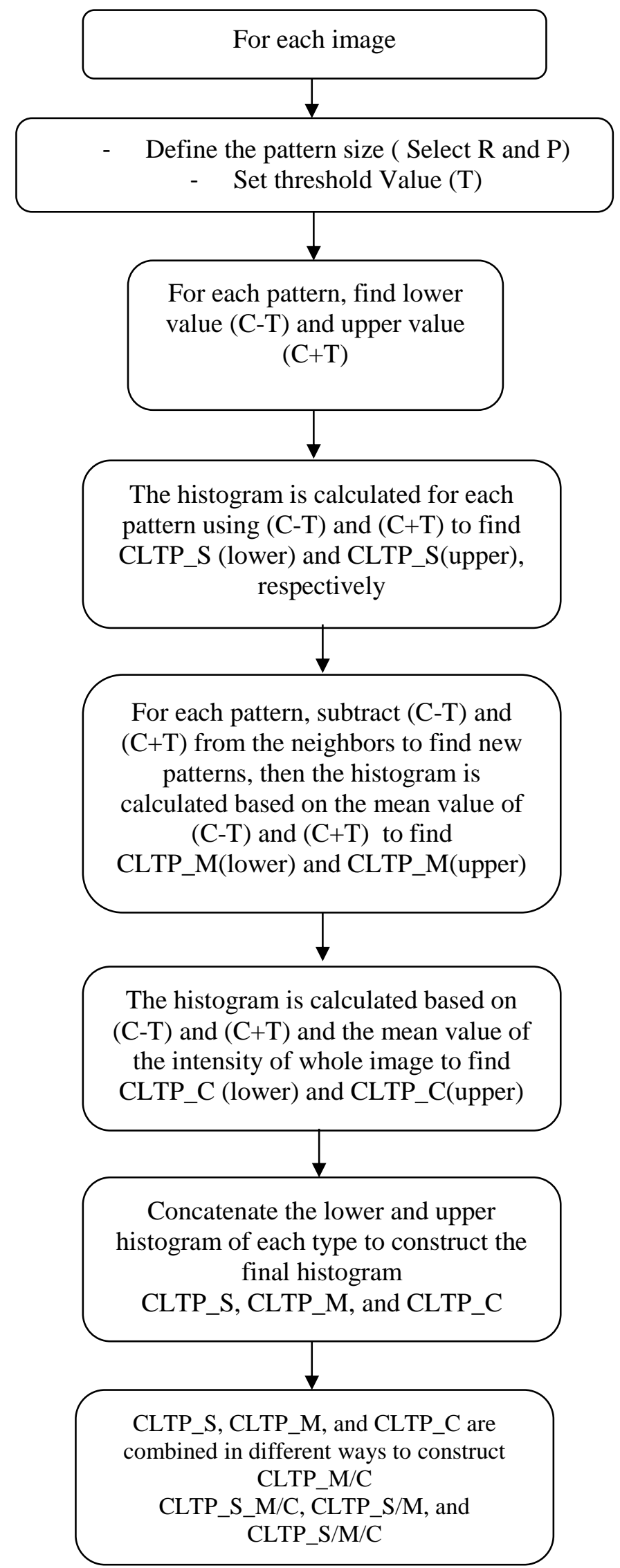

Fig. 4 Flowchart of CLTP extraction process 


\subsection{Experimental Results of CLTP noise robustness}

In this task, a special experiment was conducted on the Outex texture database (TC10) to demonstrate the effectiveness of the CLTP against the noise. Based on the experiment, the testing image was corrupted by Additive Gaussian Noise with zero mean and standard deviation that was determined according to the corresponding Signal-to-Noise Ratios (SNR) value.

Outex datasets include 16 test suites starting from Outex_TC_00010 (TC10) to Outex_TC_00016 (TC16) [23]. TC10 was used in these experiments where 480 images are used as training data and 3840 images are used as testing data. The training images are the images of "inca" illumination condition and " 0 " angle rotation while the testing images are the images under the remaining rotation angles and "inca" illumination condition. First, the testing images were corrupted by Gaussian noise. Then, the CLTP operators were extracted from the noise on three different types of texture pattern, which are $(P=8$ and $R=1),(P=16$ and $R=2)$, and $(P=24$ and $R=$ 3 ). These operators were then compared to the CLBP operators that extracted from the noisy testing images. Examples of the TC10 Outex corrupted images with different amount of Additive Gaussian Noise $(5,10,20,30$, 50, and 100) are shown in Fig. 5, while the results of the noisy experiments for different texture patterns are listed in Tables 1, 2, and 3. From Tables 1, 2, and 3, the CLTP showed more robustness against noise compared to CLBP texture descriptor. Higher classification accuracy has been achieved using the CLTP compare to CLBP with different types of SNR ratios.

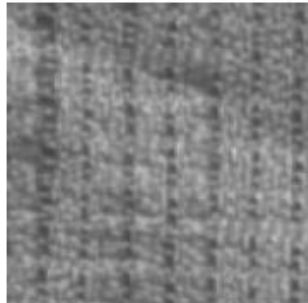

(a) SNR 100

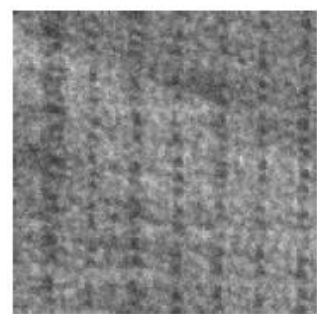

(d) SNR 20

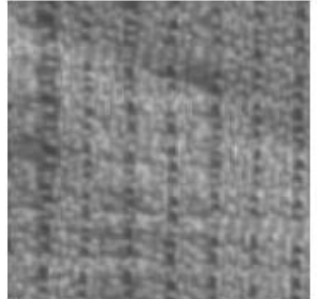

(b) SNR 50

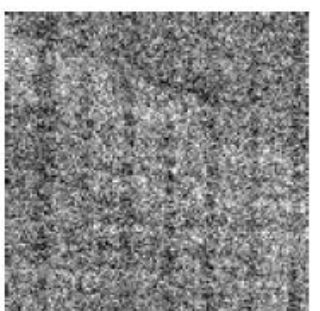

(e) SNR 10

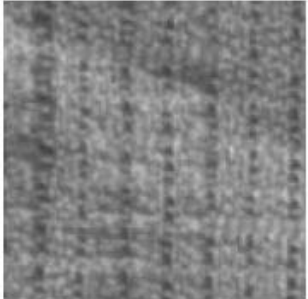

(c) SNR 30

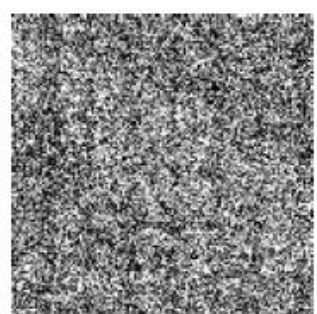

(f) SNR 5

Fig. 5: Examples of TC10 Outex dataset with Additive Gaussian Noise of different Signal-To-Noise Ratios (SNR) 
Table 1: Classification rate(\%) on TC10 Outex database with Additive Gaussian Noise of different Signal-To-Noise Ratios (SNR) when texture pattern $\mathrm{R}=3, \mathrm{P}=24$

\begin{tabular}{lrrrrrr}
\hline & SNR100 & \multicolumn{1}{c}{ SNR 50 } & \multicolumn{1}{c}{ SNR 30 } & \multicolumn{1}{c}{ SNR 20 } & \multicolumn{1}{c}{ SNR 10 } & \multicolumn{1}{c}{ SNR 5 } \\
\hline CLBP_S & 82.45 & 82.37 & 77.92 & 65.88 & 49.87 & 21.33 \\
CLTP_S & $\mathbf{9 4 . 2 2}$ & $\mathbf{9 2 . 2 4}$ & $\mathbf{8 9 . 2 2}$ & $\mathbf{8 3 . 1 2}$ & $\mathbf{6 3 . 9 8}$ & $\mathbf{3 7 . 4 2}$ \\
\hline CLBP_M & 81.66 & 80.14 & 82.16 & 66.08 & 52.16 & 33.31 \\
CLTP_M & $\mathbf{9 4 . 0 6}$ & $\mathbf{9 4 . 0 6}$ & $\mathbf{9 2 . 2 1}$ & $\mathbf{8 2 . 4 3}$ & $\mathbf{6 6 . 8 6}$ & $\mathbf{5 0 . 3 9}$ \\
\hline CLBP_M/C & 90.42 & 89.42 & 92.40 & 71.75 & 53.11 & 37.73 \\
CLTP_M/C & $\mathbf{9 5 . 8 9}$ & $\mathbf{9 5 . 5 5}$ & $\mathbf{9 4 . 8 2}$ & $\mathbf{8 6 . 4 6}$ & $\mathbf{6 8 . 6 9}$ & $\mathbf{7 0 . 8 9}$ \\
\hline CLBP_S_M/C & 94.32 & 94.30 & 93.52 & 72.19 & 53.90 & 33.23 \\
CLTP_S_M/C & $\mathbf{9 6 . 5 6}$ & $\mathbf{9 6 . 2 2}$ & $\mathbf{9 5 . 2 3}$ & $\mathbf{8 8 . 0 4}$ & $\mathbf{6 9 . 1 5}$ & $\mathbf{6 0 . 8 3}$ \\
\hline CLBP_S/M & 95.42 & 95.52 & 94.61 & 75.03 & 52.99 & 30.63 \\
CLTP_S/M & $\mathbf{9 6 . 6 4}$ & $\mathbf{9 6 . 8 2}$ & $\mathbf{9 5 . 2 1}$ & $\mathbf{8 9 . 1 1}$ & $\mathbf{7 2 . 5 6}$ & $\mathbf{6 1 . 4 1}$ \\
\hline CLBP_S/M/C & 96.55 & $\mathbf{9 7 . 5 0}$ & 97.16 & 77.64 & 56.33 & 39.30 \\
CLTP_S/M/C & $\mathbf{9 6 . 9 5}$ & 97.08 & $\mathbf{9 7 . 2 1}$ & $\mathbf{8 9 . 8 7}$ & $\mathbf{8 2 . 4 6}$ & $\mathbf{7 6 . 9 8}$ \\
\hline
\end{tabular}

Table 2: Classification rate(\%) on TC10 Outex database with Additive Gaussian Noise of different Signal-To-Noise Ratios (SNR) when texture pattern $\mathrm{R}=2, \mathrm{P}=16$

\begin{tabular}{lrlllll} 
& SNR100 & SNR 50 & SNR 30 & SNR 20 & SNR 10 & SNR 5 \\
\hline CLBP_S & 90.26 & 90.26 & 88.36 & 54.22 & 42.49 & 22.17 \\
CLTP_S & $\mathbf{9 6 . 9 8}$ & $\mathbf{9 6 . 8 5}$ & $\mathbf{9 5 . 9 9}$ & $\mathbf{5 9 . 2 2}$ & $\mathbf{5 1 . 0 3}$ & $\mathbf{3 9 . 5 0}$ \\
\hline CLBP_M & 93.67 & 86.41 & 86.22 & 53.46 & 42.87 & 29.02 \\
CLTP_M & $\mathbf{9 7 . 2 9}$ & $\mathbf{9 7 . 4 2}$ & $\mathbf{9 7 . 2 9}$ & $\mathbf{6 9 . 4 8}$ & $\mathbf{5 3 . 9 1}$ & $\mathbf{5 1 . 3 2}$ \\
\hline CLBP_M/C & 96.98 & 94.45 & 89.06 & 63.78 & 55.22 & 31.55 \\
CLTP_M/C & $\mathbf{9 7 . 9 2}$ & $\mathbf{9 8 . 1 3}$ & $\mathbf{9 7 . 9 2}$ & $\mathbf{8 5 . 1 8}$ & $\mathbf{6 5 . 4 7}$ & $\mathbf{5 8 . 4 0}$ \\
\hline CLBP_S_M/C & 97.48 & 96.51 & 90.17 & 60.08 & 54.87 & 34.70 \\
CLTP_S_M/C & $\mathbf{9 8 . 4 9}$ & $\mathbf{9 8 . 3 9}$ & $\mathbf{9 8 . 0 5}$ & $\mathbf{7 9 . 2 2}$ & $\mathbf{7 0 . 4 3}$ & $\mathbf{6 0 . 0 1}$ \\
\hline CLBP_S/M & 96.94 & 97.76 & 91.00 & 59.43 & 56.81 & 35.44 \\
CLTP_S/M & $\mathbf{9 7 . 8 1}$ & $\mathbf{9 7 . 9 9}$ & $\mathbf{9 7 . 7 6}$ & $\mathbf{8 4 . 9 2}$ & $\mathbf{7 5 . 1 4}$ & $\mathbf{6 2 . 5 3}$ \\
\hline CLBP_S/M/C & 98.91 & 98.96 & 92.89 & 68.01 & 58.08 & 41.78 \\
CLTP_S/M/C & $\mathbf{9 8 . 9 6}$ & $\mathbf{9 8 . 8 3}$ & $\mathbf{9 8 . 7 2}$ & $\mathbf{9 2 . 0 1}$ & $\mathbf{8 0 . 7 6}$ & $\mathbf{6 8 . 1 1}$ \\
\hline
\end{tabular}


Table 3: Classification rate(\%) on TC10 Outex database with Additive Gaussian Noise of different Signal-To-Noise Ratios (SNR) when texture pattern $\mathrm{R}=1, \mathrm{P}=8$

\begin{tabular}{lllllll} 
CLBP_S & 95.82 & 89.95 & 89.22 & 66.43 & 46.68 & 19.66 \\
CLTP_S & $\mathbf{9 8 . 2 3}$ & $\mathbf{9 8 . 1 3}$ & $\mathbf{9 7 . 5 0}$ & $\mathbf{7 4 . 8 7}$ & $\mathbf{7 3 . 5 5}$ & $\mathbf{3 5 . 7 2}$ \\
\hline CLBP_M & 95.86 & 92.86 & 92.55 & 63.15 & 49.19 & 25.99 \\
CLTP_M & $\mathbf{9 7 . 9 9}$ & $\mathbf{9 8 . 0 7}$ & $\mathbf{9 7 . 9 4}$ & $\mathbf{8 5 . 6 8}$ & $\mathbf{7 5 . 8 7}$ & $\mathbf{4 8 . 1 2}$ \\
\hline CLBP_M/C & 97.60 & 97.86 & 97.76 & 77.58 & 52.45 & 29.79 \\
CLTP_M/C & $\mathbf{9 8 . 5 4}$ & $\mathbf{9 8 . 5 7}$ & $\mathbf{9 8 . 3 9}$ & $\mathbf{9 5 . 0 0}$ & $\mathbf{7 7 . 1 2}$ & $\mathbf{5 3 . 8 0}$ \\
\hline CLBP_S_M/C & 97.94 & 97.94 & 97.73 & 72.40 & 58.41 & 30.76 \\
CLTP_S_M/C & $\mathbf{9 8 . 9 8}$ & $\mathbf{9 8 . 8 5}$ & $\mathbf{9 8 . 7 8}$ & $\mathbf{9 5 . 3 6}$ & $\mathbf{7 7 . 9 9}$ & $\mathbf{5 4 . 8 5}$ \\
\hline CLBP_S/M & $\mathbf{9 9 . 1 9}$ & 98.96 & 97.75 & 72.40 & 58.41 & 30.76 \\
CLTP_S/M & 99.04 & $\mathbf{9 9 . 0 4}$ & $\mathbf{9 8 . 9 8}$ & $\mathbf{9 6 . 8 5}$ & $\mathbf{7 9 . 5 4}$ & $\mathbf{5 5 . 7 0}$ \\
\hline CLBP_S/M/C & 98.70 & 98.88 & 98.01 & 81.33 & 60.33 & 34.09 \\
CLTP_S/M/C & $\mathbf{9 9 . 1 7}$ & $\mathbf{9 9 . 1 7}$ & $\mathbf{9 9 . 1 7}$ & $\mathbf{9 8 . 2 8}$ & $\mathbf{8 5 . 7 2}$ & $\mathbf{6 6 . 4 6}$ \\
\hline
\end{tabular}

\subsection{Experimental Results for Image Category Recognition}

\subsubsection{Experimental Results on OT8 Scene Dataset}
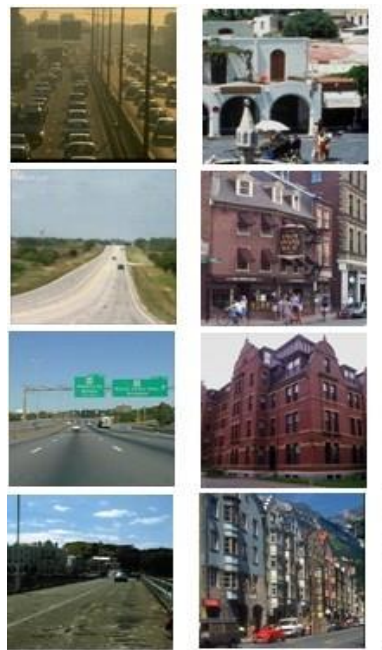

a) OT Man-Made images (columns from left to right; highway, inside city, street and tall building)

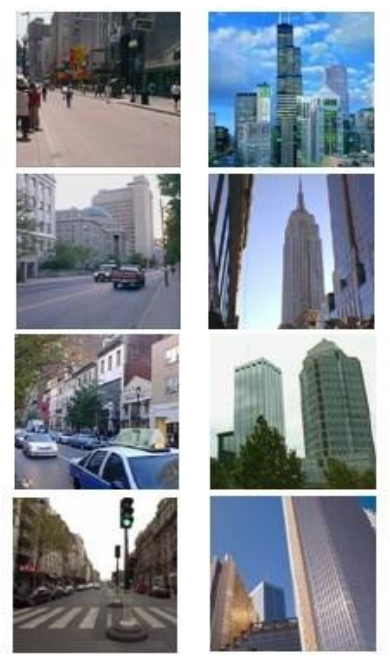$$
\text { tall }
$$
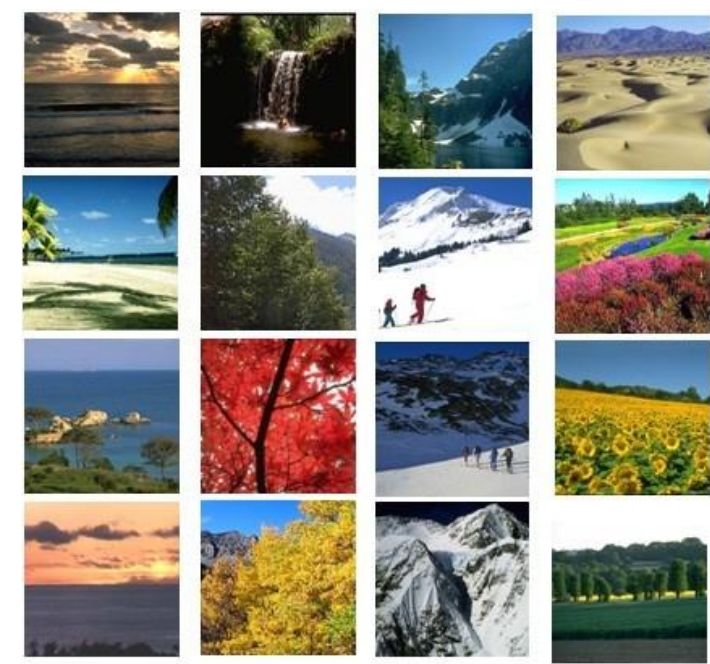

b) OT Natural images (columns from left to right; coast, forest, mountain and open city)

Fig. 6: Some images from Oliva \& Torralba (OT) dataset.

Oliva \& Torralba dataset (OT8) is 2688 color images in total [17]. OT8 contains eight categories; coast, forest, mountain, open country, highway, insidecity, tallbuilding, and street. These images are in JPG format and 
contain average size of $265 \times 265$ pixels. Some images of the OT8 dataset are shown in Fig. 6 . In this experiment, the OT8 scene dataset was used to evaluate the proposed CLTP and compared its performance with the grey CLBP under various numbers of training images. All CLTP operators were extracted on three different types of texture pattern, which are $(\mathrm{P}=8, \mathrm{R}=1),(\mathrm{P}=16, \mathrm{R}=2)$, and $(\mathrm{P}=24, \mathrm{R}=3)$ and compared to the CLBP operators. The final classification accuracy was determined by the average percentage over a hundred random splits. The OT8 average classification results for $N=(10,30,40,50,100,150,200)$ are shown in Table 4. In each class, $N$ images were used for training while the remaining images in the class were used for testing.

Aside from CLTP_S/M operator when $N=(100,150,200)$ at $P=8, R=1$, all CLTP operators have achieved higher performance than CLBP operators for all $N$ numbers of training images at radiuses 1,2 and 3 . Thus, it has proved the priority of the CLTP. The best classification accuracy was obtained by $C L T P \_S / M / C_{2,16}$ operator, which has reached up to $57.63 \%$ while the $C L B P \_S / M / C_{1,8}$ has achieved the best classification accuracy, which has reached up to $55.83 \%$.

\subsubsection{Experimental Results on Event Sport Dataset}
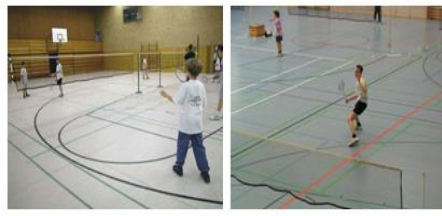

(a) Badminton
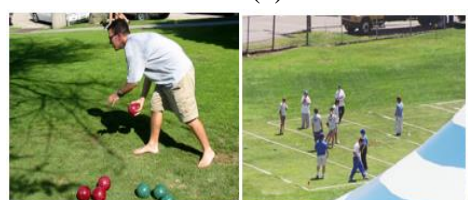

(b) Bocce

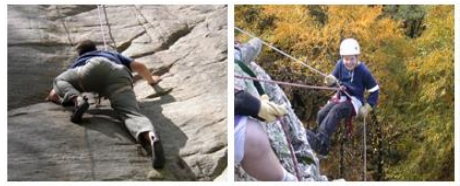

(c) RockClimbing

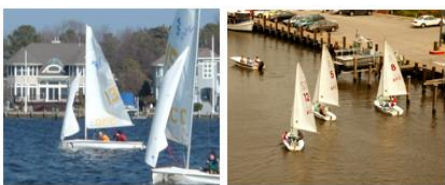

(d) Sailing
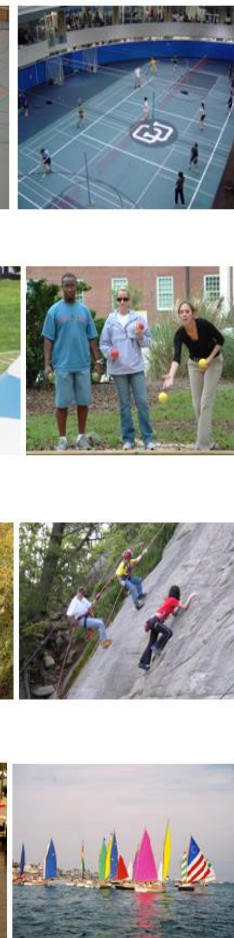

Fig. 7: Some images from Event sport dataset.
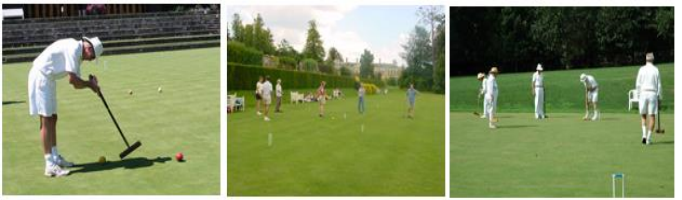

(e) Croquet
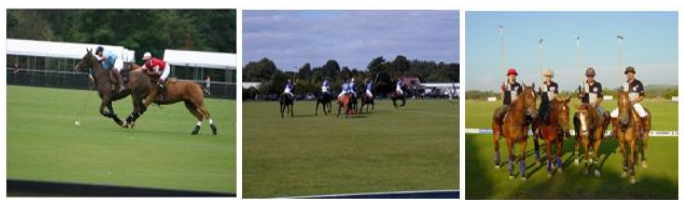

(f) Polo
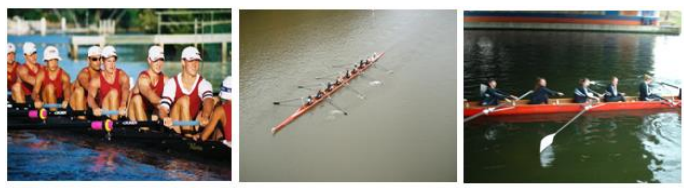

(g) Rowing
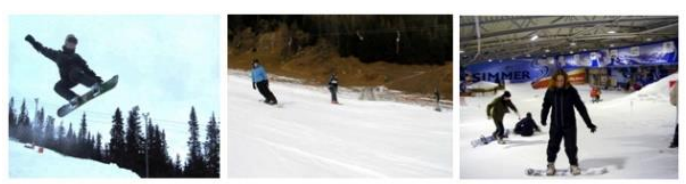

(h) Snowboarding

In this experiment, the Event sport dataset was used to evaluate the proposed CLTP and compared its performance with the grey CLBP under various numbers of training images. The Event sport dataset has eight categories, namely, rowing, badminton, polo, bocce, snow boarding, croquet, sailing, and rock climbing [18]. Fig. 7 shows some examples of the Event sport images. Similar to the OT8 experiments, three different types of texture pattern were used, which are $(\mathrm{P}=8$ and $\mathrm{R}=1),(\mathrm{P}=16$ and $\mathrm{R}=2)$, and $(\mathrm{P}=24$ and $\mathrm{R}=3)$. In each class of Event dataset, $N$ images were used for training while the remaining images in the class were used for testing. The Event average classification results for $N=(5,10,20,30,40,50,60)$ are shown in Table 5. The final classification accuracy was determined by the average percentage over a hundred random splits. 
Table 4: Classification rates (\%) on OT8 dataset

\begin{tabular}{|c|c|c|c|c|c|c|c|c|c|c|c|c|c|c|c|c|c|c|c|c|c|}
\hline & \multicolumn{7}{|c|}{$\mathrm{R}=1, \mathrm{P}=8$} & \multicolumn{7}{|c|}{$\mathrm{R}=2, \mathrm{P}=16$} & \multicolumn{7}{|c|}{$\mathrm{R}=3, \mathrm{P}=24$} \\
\hline & 10 & 30 & 40 & 50 & 100 & 150 & 200 & 10 & 30 & 40 & 50 & 100 & 150 & 200 & 10 & 30 & 40 & 50 & 100 & 150 & 200 \\
\hline CLBP_S & 32.67 & 37.69 & 38.80 & 39.30 & 41.49 & 42.43 & 42.87 & 34.64 & 39.11 & 40.05 & 41.34 & 43.89 & 44.92 & 45.58 & 34.21 & 39.33 & 40.69 & 41.38 & 43.69 & 44.61 & 44.57 \\
\hline CLTP_S & 42.98 & 47.06 & 48.65 & 48.82 & 51.75 & 52.28 & 52.94 & 43.98 & 47.75 & 49.07 & 49.39 & 52.01 & 51.81 & 52.94 & 44.25 & 46.24 & 47.47 & 48.25 & 50.85 & 51.81 & 52.94 \\
\hline CLBP_M & 39.03 & 42.59 & 43.35 & 43.87 & 45.49 & 46.01 & 45.72 & 40.15 & 43.28 & 44.08 & 44.56 & 46.48 & 47.22 & 46.98 & 38.50 & 42.27 & 43.29 & 43.75 & 45.41 & 45.86 & 45.60 \\
\hline $\mathrm{CLTP}_{-} \mathrm{M}$ & 41.30 & 46.57 & 46.75 & 46.20 & 47.93 & 49.73 & 50.37 & 44.36 & 47.88 & 48.90 & 49.13 & 50.26 & 50.87 & 52.21 & 43.75 & 46.00 & 47.34 & 48.03 & 50.37 & 50.94 & 50.64 \\
\hline CLBP_M/C & 38.99 & 43.35 & 44.43 & 45.26 & 47.46 & 48.42 & 48.35 & 38.53 & 43.28 & 44.23 & 45.05 & 47.66 & 48.83 & 49.19 & 37.78 & 41.99 & 43.01 & 43.90 & 46.14 & 47.32 & 47.55 \\
\hline CLBP_S_M/C & 40.28 & 45.14 & 46.25 & 47.26 & 49.96 & 51.11 & 51.54 & 39.76 & 45.14 & 46.29 & 47.33 & 49.91 & 51.31 & 51.52 & 39.83 & 44.32 & 45.51 & 46.43 & 48.78 & 49.57 & 49.74 \\
\hline $\mathrm{CLTP}_{-} \mathrm{S}_{-} \mathrm{M} / \mathrm{C}$ & 46.01 & 49.55 & 50.17 & 51.44 & 53.28 & 54.10 & 54.78 & 46.59 & 49.75 & 50.97 & 52.49 & 54.03 & 55.38 & 55.79 & 45.44 & 48.73 & 50.59 & 50.96 & 53.34 & 54.30 & $\mathbf{5 4 . 5 0}$ \\
\hline CLBP_S/M & 42.43 & 47.85 & 49.28 & 50.22 & 53.05 & 54.37 & 54.65 & 42.71 & 47.98 & 49.37 & 50.42 & 52.76 & 53.87 & 54.25 & 42.18 & 47.18 & 48.71 & 49.49 & 51.70 & 52.47 & 52.78 \\
\hline CLTP_S/M & 46.09 & 48.33 & 50.17 & 51.44 & 52.60 & 53.90 & 54.04 & 46.66 & 48.98 & 51.39 & 51.49 & 53.76 & $\mathbf{5 4 . 5 7}$ & 54.96 & 45.82 & 48.57 & 49.75 & 51.18 & 53.18 & 55.11 & 55.51 \\
\hline CLBP_S/M/C & 43.18 & 48.47 & 49.94 & 51.04 & 53.81 & 55.27 & 55.83 & 42.48 & 48.49 & 49.70 & 50.46 & 53.25 & 54.53 & 54.85 & 42.01 & 47.19 & 48.40 & 49.31 & 52.14 & 53.42 & 53.61 \\
\hline CLTP_S/M/C & 48.24 & 50.12 & 51.69 & 52.49 & 55.40 & 56.32 & 57.26 & 48.89 & 51.80 & 52.03 & 53.19 & 55.72 & 57.06 & 57.63 & 48.66 & 50.33 & 51.14 & 52.62 & 55.46 & 55.44 & 56.62 \\
\hline
\end{tabular}


Table 5: Classification rates (\%) on Event sport dataset

\begin{tabular}{|c|c|c|c|c|c|c|c|c|c|c|c|c|c|c|c|c|c|c|c|c|c|}
\hline & \multicolumn{7}{|c|}{$\mathrm{R}=1, \mathrm{P}=8$} & \multicolumn{7}{|c|}{$\mathrm{R}=2, \mathrm{P}=16$} & \multicolumn{7}{|c|}{$\mathrm{R}=3, \mathrm{P}=24$} \\
\hline & 5 & 10 & 20 & 30 & 40 & 50 & 60 & 5 & 10 & 20 & 30 & 40 & 50 & 60 & 5 & 10 & 20 & 30 & 40 & 50 & 60 \\
\hline CLBP $\mathrm{S}$ & 25.01 & 29.88 & 34.74 & 38.26 & 40.14 & 41.72 & 43.08 & 24.11 & 28.07 & 32.73 & 35.88 & 37.98 & 39.51 & 41.09 & 23.00 & 27.22 & 31.27 & 34.04 & 36.26 & 37.92 & 39.29 \\
\hline CLTP_S & 28.20 & 33.02 & 36.65 & 37.57 & 39.24 & 41.22 & 42.04 & 32.03 & 33.62 & 35.80 & 38.91 & 40.98 & 42.24 & 43.13 & 31.16 & 35.07 & 37.48 & 38.98 & 40.75 & 42.50 & 43.69 \\
\hline CLBP $\mathrm{M}$ & 24.41 & 28.33 & 33.00 & 36.04 & 38.03 & 39.86 & 41.26 & 25.23 & 29.97 & 35.07 & 38.01 & 39.93 & 41.69 & 43.03 & 26.29 & 30.62 & 35.21 & 37.93 & 39.89 & 41.54 & 42.62 \\
\hline CLTP_M & 27.42 & 31.89 & 34.53 & 37.04 & 39.00 & 40.12 & 42.68 & 30.67 & 32.15 & 36.01 & 39.13 & 41.62 & 42.24 & 43.40 & 31.36 & 33.27 & 36.42 & 39.66 & 41.15 & 42.84 & 44.24 \\
\hline CLBP_M/C & 24.47 & 27.95 & 32.36 & 35.28 & 37.57 & 39.23 & 40.69 & 25.77 & 29.58 & 33.85 & 36.91 & 38.84 & 40.70 & 42.17 & 26.39 & 29.85 & 34.10 & 37.36 & 38.90 & 40.48 & 41.95 \\
\hline CLTP_M/C & 29.04 & 31.82 & 37.14 & 38.24 & 42.57 & 43.00 & 45.40 & 31.06 & 33.36 & 37.77 & 41.22 & 44.48 & 45.46 & 46.77 & 30.44 & 36.08 & 38.83 & 42.95 & 44.18 & 46.76 & 47.35 \\
\hline CLBP_S_M/C & 26.33 & 30.80 & 35.80 & 39.21 & 42.11 & 43.55 & 45.34 & 27.16 & 31.55 & 36.68 & 39.88 & 42.16 & 44.12 & 45.70 & 27.30 & 31.60 & 36.41 & 39.90 & 41.91 & 43.79 & 45.10 \\
\hline CLTP_S_M/C & 30.86 & 34.02 & 38.55 & 42.05 & 43.53 & 46.56 & 47.95 & 33.66 & 37.16 & 42.49 & 43.99 & 45.91 & 47.75 & 50.86 & 32.99 & 36.88 & 41.80 & 43.93 & 46.09 & 47.61 & 48.99 \\
\hline CLBP_S/M & 27.56 & 32.35 & 38.42 & 41.76 & 44.07 & 46.02 & 47.76 & 28.14 & 33.69 & 38.65 & 42.11 & 44.20 & 46.54 & 47.99 & 28.43 & 33.08 & 38.22 & 41.30 & 43.34 & 45.59 & 47.01 \\
\hline CLTP_S/M & 30.15 & 33.62 & 37.49 & 40.63 & 42.73 & 44.78 & 45.50 & 34.83 & 36.36 & 39.75 & 42.20 & 44.96 & 45.72 & 47.68 & 32.53 & 36.55 & 39.46 & 42.35 & 46.01 & 47.44 & 47.07 \\
\hline CLBP_S/M/C & 28.47 & 33.22 & 38.67 & 42.42 & 44.97 & 47.16 & 48.86 & 29.30 & 34.15 & 40.17 & 43.31 & 46.17 & 48.26 & 49.85 & 28.80 & 33.94 & 39.60 & 43.04 & 45.58 & 47.67 & 49.24 \\
\hline CLTP_S/M/C & 30.99 & 35.42 & 40.24 & 43.39 & 45.83 & 46.82 & 48.50 & 33.07 & 38.63 & 42.21 & 46.98 & 47.42 & 49.62 & 50.96 & 34.55 & 38.96 & 42.86 & 45.73 & 47.53 & 50.85 & 51.37 \\
\hline
\end{tabular}


Other than $C L T P \_S_{1,8}, C L T P \_S / M_{1,8}, C L T P \_S / M_{2,16}$, and $C L T P \_S / M / C_{1,8}$ operators, all CLTP operators have achieved higher performance than CLBP operators. The performances of $C L B P \_S_{1,8}$ when $N=(30,40,50,60)$, $C L B P \_S / M_{1,8}$ when $N=(20,30,40,50,60), C L T P \_S / M_{2,16}$ when $N=(50,60)$, and $C L T P \_S / M / C_{l, 8}$ when $N=$ $(50,60)$, have outperformed the corresponding CLTP operators. From these experiments, it is found that CLTP operators perform better than CLBP operators. The best classification accuracy was obtained by $C L T P \_S / M / C_{3,24}$ operator which has reached up to $51.37 \%$ while $C L B P \_S / M / C_{2,16}$ has achieved the best classification accuracy, with $49.85 \%$.

\subsubsection{Experimental Results on 2D HeLa Medical Image}

The automated identification of medical images and the automated medical diagnostics have become important fields in the research on computer vision and image processing. In this section, the 2D HeLa dataset images were used to evaluate the proposed CLTP texture descriptor. The 2D HeLa images contain HeLa cells stained with various organelle-specific fluorescent dyes.
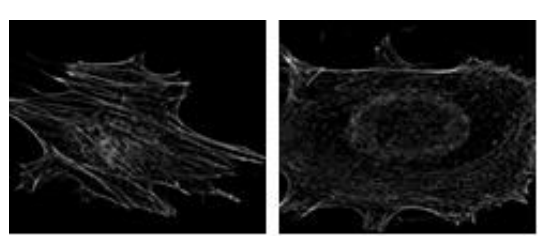

(a) Action
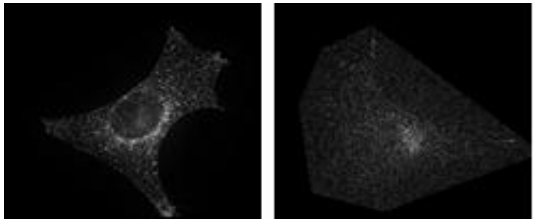

(c) Endsome
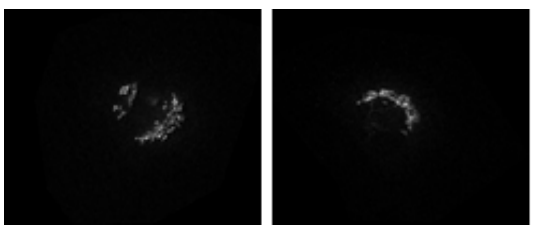

(e) Golgia Giantin
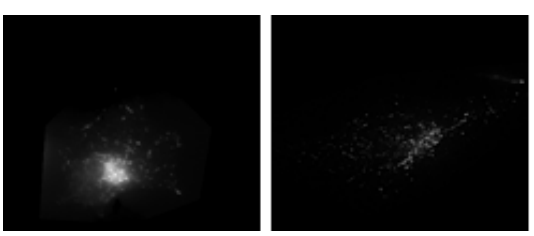

(g) Lysosome
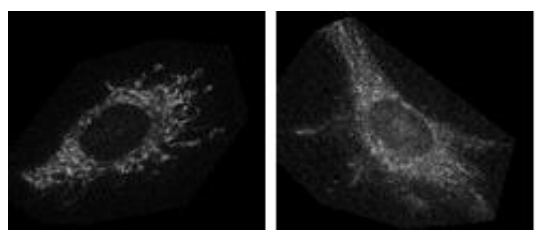

(i) Mitochondria
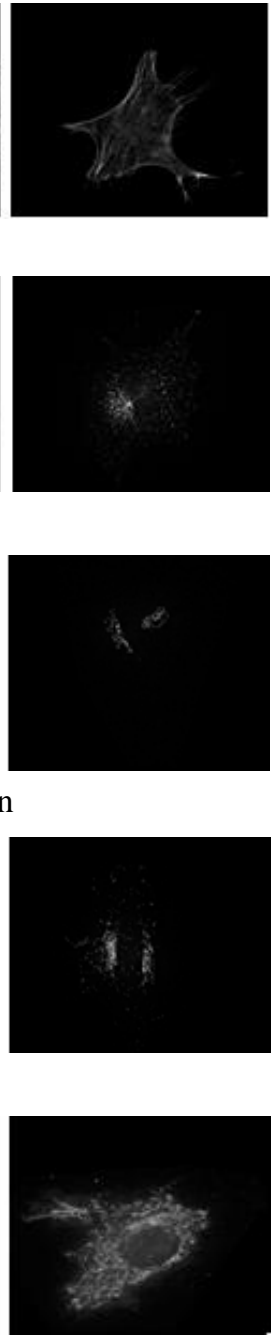

Fig. 8: Some images from 2D HeLa dataset.
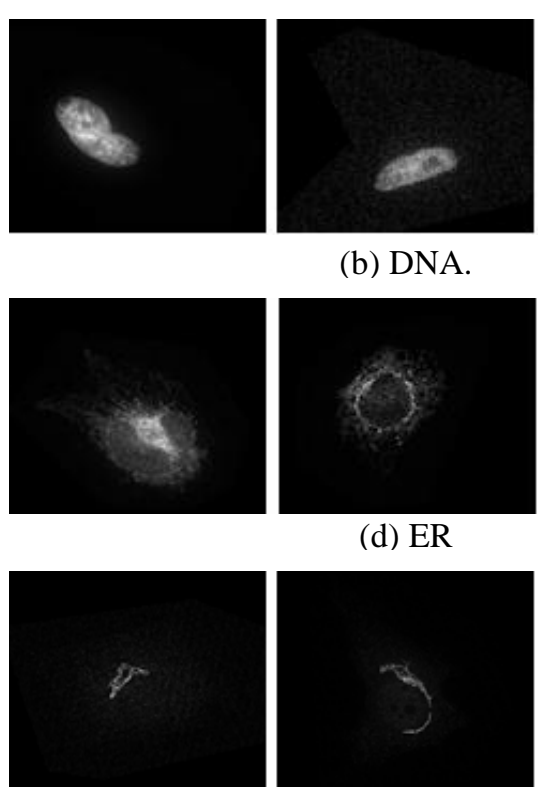

(f) Golgi GPP130
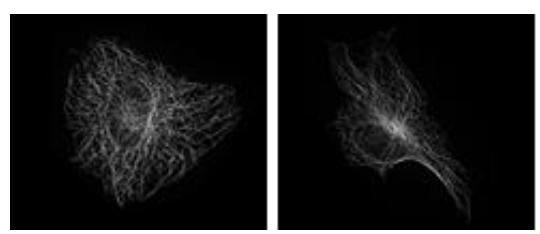

(h) Microtubules
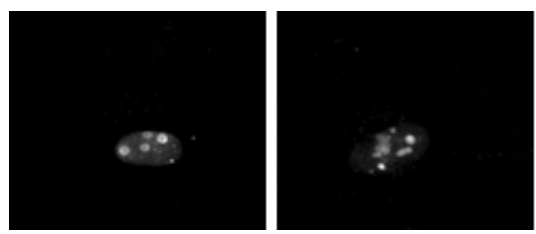

(b) DNA

(d) ER

(j) Nucleolus
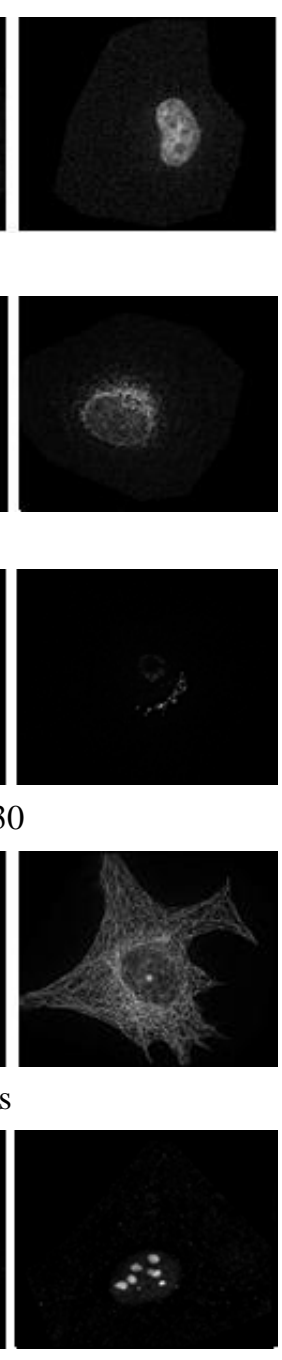

(j) Nucleolus

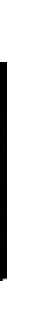

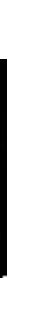

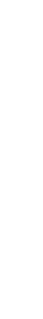


Some images of 2D HeLa dataset are shown in Fig. 8. The automatic classification and identification of subcellular organelles help in the discovery of new genes or unknown function genes.In addition, fluorescently tagging the protein produced by any given gene is possible. As shown in Fig. 8, differentiating and distinguishing classes of 2D HeLa dataset, such as Endosomes and Lysosomes, and Golgia Giantin and Golgi GPP130 may be difficult. Many researchers used and considered this classification problem [19]-[21].

In this experiment, the 2D HeLa image dataset was evaluated using the proposed CLTP texture descriptor. Similar to [21], 4/5 of the images in each class were randomly selected as training images and the remaining $1 / 5$ were used as testing images. The final classification accuracy is the average percentage over 10 random splits. The $\chi 2$ SVM classifier was used in this experiment. Four types of texture pattern radii, $(\mathrm{R}=1,2,3$, and 5) with three different neighbour sizes $(\mathrm{P}=8,16$ and $\mathrm{P}=24)$ were used to extract the CLTP texture operators.

Table 6: Classification rates (\%) on 2D Hela dataset.

\begin{tabular}{|c|c|c|c|c|}
\hline & $R=1, P=8$ & $R=2, P=8$ & $R=3, P=8$ & $R=5, P=8$ \\
\hline CLTP_S & 84.57 & 90.74 & 91.98 & 83.95 \\
\hline CLTP_M & 79.63 & 87.65 & 87.04 & 87.04 \\
\hline CLTP_M/C & 89.51 & 90.12 & 88.27 & 88.89 \\
\hline CLTP_S_M/C & 93.21 & 93.83 & 90.12 & 90.12 \\
\hline CLTP_S/M & 94.44 & 93.21 & 94.44 & 93.83 \\
\hline \multirow[t]{2}{*}{ CLTP_S/M/C } & 88.89 & 94.44 & 91.98 & 91.36 \\
\hline & $R=1, P=16$ & $R=2, P=16$ & $R=3, P=16$ & $R=5, P=16$ \\
\hline CLTP_S & 88.89 & 91.98 & 93.83 & 88.89 \\
\hline CLTP_M & 88.27 & 91.98 & 87.04 & 87.04 \\
\hline CLTP_M/C & 86.42 & 90.12 & 88.27 & 91.36 \\
\hline CLTP_S_M/C & 90.74 & 91.36 & 91.36 & 88.89 \\
\hline CLTP_S/M & 88.27 & 95.62 & 92.59 & 92.59 \\
\hline \multirow[t]{2}{*}{ CLTP_S/M/C } & 90.74 & 92.59 & 92.59 & 93.21 \\
\hline & $R=1, P=24$ & $R=2, P=24$ & $R=3, P=24$ & $R=5, P=24$ \\
\hline CLTP_S & 88.89 & 93.83 & 93.21 & 92.59 \\
\hline CLTP_M & 82.10 & 90.12 & 90.12 & 88.89 \\
\hline CLTP_M/C & 85.19 & 88.89 & 90.12 & 91.36 \\
\hline CLTP_S_M/C & 87.65 & 91.36 & 92.59 & 92.59 \\
\hline CLTP_S/M & 90.74 & 91.98 & 91.98 & 93.21 \\
\hline CLTP_S/M/C & 88.89 & 93.83 & 90.74 & 90.74 \\
\hline
\end{tabular}

As shown in Table 6, the best classification rate was obtained using CLTP_S/M $M_{2,16}$, with $95.62 \%$. For comparison, experiments with the same setup were conducted using the CLBP texture operators. The $C L B P \_S / M / C_{3,8}$, with $94.44 \%$, obtained the best accuracy rate using the CLBP texture operators. Table 6 summaries the best obtained results with some state-of-the art results on the $2 \mathrm{D}$ HeLa dataset. 
Table 7: Results of the state-of-the-art using 2D Hela dataset.

\begin{tabular}{ccc}
\multicolumn{2}{c}{ Description } & Accuracy \\
\hline$C L T P_{-} S / M 2,16$ (This study) & $\mathbf{9 5 . 6 2}$ \\
$C L B P \_S / M / C 3,8$ & (This study) & 94.44 \\
$E Q P \beta$ & {$[20]$} & 92.4 \\
DomLT P & {$[21]$} & 92.2 \\
Chebira et al. & {$[22]$} & 95.3 \\
\hline
\end{tabular}

\subsubsection{Experimental Results on USM Scene Dataset}

USM dataset is a new scene dataset where the images in the dataset are collected by the Centre for Instructional Technology and Multimedia, Universiti Sains Malaysia. A NIKON D70s camera was used to capture each image in a 24 bit RGB format and $1488 * 2240$ resolutions.
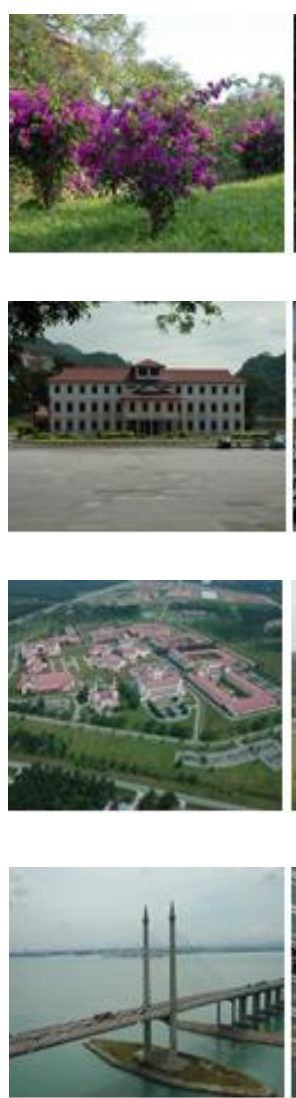
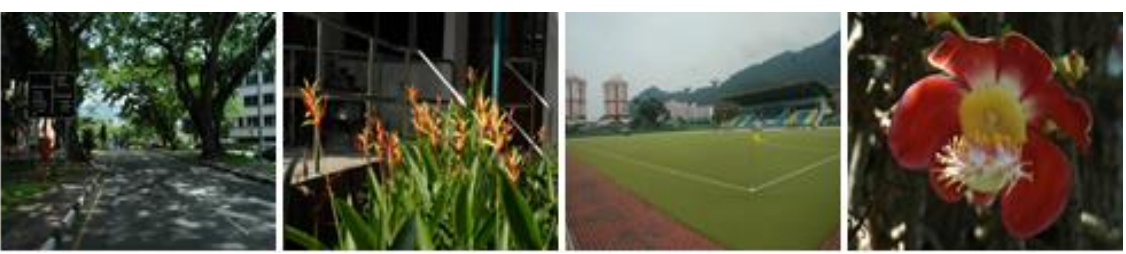

(a) USM natural category
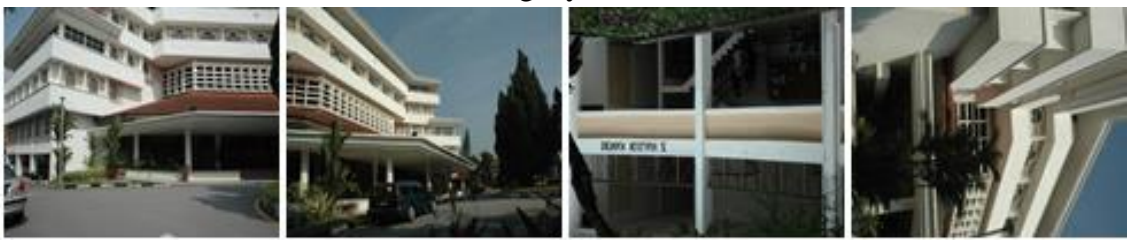

(b) USM building category
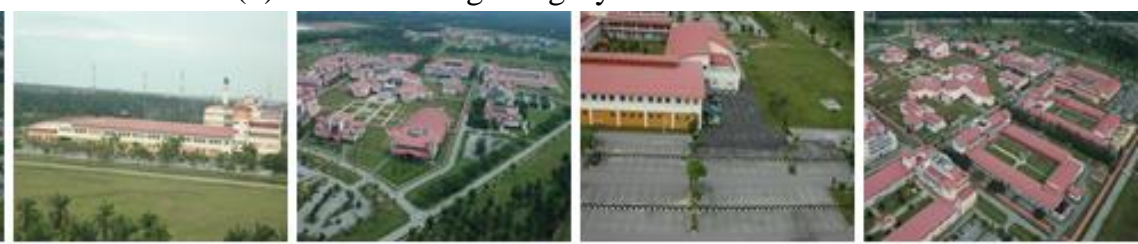

(c) USM engineering category
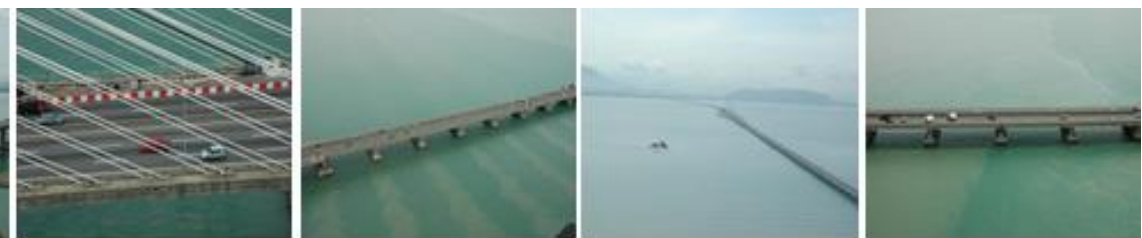

(d) USM bridge categorv

Fig. 9: Some images from USM scene dataset.

This dataset can be considered as the first version where the images are classified into four categories. These categories are: USM natural category which has 77 images, USM building category with 131 images, USM engineering category which has 128 images, and USM bridge category with 54 images. For this experiment, the resolution of the images is decreased to $512 * 512$. The inter-class variability and intra-class variability challenges also appeared in the USM scene dataset. Some of the USM dataset images are shown Fig. 9. In this experiment, three different texture patterns, $[(\mathrm{R}=1, \mathrm{P}=8),(\mathrm{R}=2, \mathrm{P}=16)$ and $(\mathrm{R}=3, \mathrm{P}=24)]$ were used to extract the CLTP texture operators. In each class, $N$ number of images was used for training, whereas the remaining images were used for testing. The final classification accuracy is the average percentage over 10 random splits. The USM scene dataset 
average classification rates for $N=(5,10,20,30,40)$ are shown in Table 8 where the best classification accuracy rate was obtained by the $C L T P \_S / M_{3,24}$ operator, with $93.91 \%$, followed by $C L T P \_S / M / C_{3,24}$ operator, with $93.04 \%$. For comparison, this experiment was repeated using the CLBP texture operators under the same setup. The classification accuracy rates obtained via CLBP operators are shown in Table 9 which indicates the superiority of CLTP over CLBP texture operators. The best classification rate obtained via CLBP texture operators did not exceed the $87.24 \%$ achieved using $C L B P \_S / M_{2,16}$ operator.

Table 8: Classification rates (\%) on USM scene dataset using CLTP texture operators.

\begin{tabular}{c|ccccc|cccccc|ccccc}
\hline \multirow{2}{*}{ Description } & \multicolumn{4}{|c|}{$\mathrm{R}=1, \mathrm{P}=8$} & \multicolumn{5}{c|}{$\mathrm{R}=2, \mathrm{P}=16$} & \multicolumn{5}{c}{$\mathrm{R}=3, \mathrm{P}=24$} \\
\cline { 2 - 16 } & 5 & 10 & 20 & 30 & 40 & 5 & 10 & 20 & 30 & 40 & 5 & 10 & 20 & 30 & 40 \\
\hline CLTP_S & 69.46 & 75.43 & 79.03 & 81.48 & 83.91 & 75.41 & 82.29 & 84.84 & 88.15 & 91.74 & 78.38 & 85.14 & 89.03 & 91.11 & 92.17 \\
CLTP_M & 72.70 & 75.71 & 76.45 & 79.26 & 80.87 & 75.14 & 79.43 & 83.87 & 86.67 & 87.83 & 75.41 & 79.71 & 82.58 & 86.67 & 88.70 \\
CLTP_M/C & 73.51 & 77.43 & 80.00 & 82.22 & 84.78 & 74.05 & 78.57 & 83.87 & 85.56 & 88.70 & 72.97 & 78.29 & 82.58 & 85.19 & 86.52 \\
CLTP_S_M/C & 75.95 & 78.86 & 82.58 & 87.78 & 88.26 & 77.30 & 82.57 & 86.13 & 88.15 & 90.43 & 79.73 & 81.14 & 86.13 & 89.26 & 91.74 \\
CLTP_S/M & 76.49 & 78.57 & 83.87 & 85.19 & 88.26 & 79.19 & 84.29 & 88.71 & 89.26 & 90.43 & 81.35 & 85.43 & 89.68 & 91.85 & $\mathbf{9 3 . 9 1}$ \\
CLTP_S/M/C & 76.76 & 82.29 & 86.46 & 87.41 & 89.57 & 80.27 & 83.71 & 88.06 & 90.00 & 92.61 & 82.70 & 86.86 & 90.65 & 91.48 & 93.04 \\
\hline
\end{tabular}

Table 9: Classification rates (\%) on USM scene dataset using CLBP texture operators.

\begin{tabular}{c|ccccc|cccccc|cccc}
\hline \multirow{2}{*}{ Description } & \multicolumn{4}{|c|}{$\mathrm{R}=1, \mathrm{P}=8$} & \multicolumn{4}{c|}{$\mathrm{R}=2, \mathrm{P}=16$} & \multicolumn{4}{c}{$\mathrm{R}=3, \mathrm{P}=24$} \\
\cline { 2 - 15 } & 5 & 10 & 20 & 30 & 40 & 5 & 10 & 20 & 30 & 40 & 5 & 10 & 20 & 30 & 40 \\
\hline CLBP_S & 67.87 & 73.31 & 77.25 & 79.33 & 79.91 & 68.53 & 74.35 & 79.00 & 80.96 & 82.89 & 63.68 & 71.70 & 77.52 & 80.66 & 82.27 \\
CLBP_M & 59.26 & 64.74 & 69.35 & 71.74 & 74.00 & 61.02 & 67.40 & 72.69 & 75.77 & 76.88 & 60.49 & 65.73 & 71.28 & 73.94 & 76.79 \\
CLBP_M/C & 62.74 & 67.88 & 73.43 & 75.91 & 77.89 & 61.16 & 67.99 & 73.95 & 76.67 & 79.38 & 61.49 & 67.70 & 74.45 & 77.04 & 79.50 \\
CLBP_S_M/C & 66.92 & 73.37 & 78.31 & 80.54 & 82.10 & 65.97 & 72.65 & 78.54 & 80.81 & 82.48 & 64.73 & 70.95 & 77.45 & 79.56 & 81.78 \\
CLBP_S/M & 69.87 & 76.01 & 82.38 & 85.20 & 86.57 & 70.42 & 77.18 & 82.62 & 85.73 & $\mathbf{8 7 . 2 4}$ & 67.66 & 74.73 & 80.55 & 83.97 & 86.02 \\
CLBP_S/M/C & 68.59 & 75.68 & 81.37 & 83.90 & 85.87 & 69.51 & 76.45 & 82.40 & 85.19 & 87.05 & 66.76 & 74.30 & 80.55 & 84.07 & 86.09 \\
\hline
\end{tabular}

\subsection{CONCLUSIONS}

The Completed Local Ternary Pattern (CLTP) texture descriptor was proposed to overcome the weaknesses of the Local Binary Pattern (LBP) and many of its variants descriptors in order to increase its discriminating property. Previously, the CLTP performance is evaluated for texture image classification only. However, in this paper the experiments are performed using different standard image datasets. These datasets are scene, event, medical image datasets. The purpose of this study is prove the ability of the proposed CLTP to distinguish between these datasets as well as texture datasets. Firstly, the performance of the CLTP descriptor against the noise was evaluated. The CLTP has shown high robustness against the noise as compared to CLBP. Then, the performances of CLTP was evaluated and investigated for image category recognition. The CLTP performance was analysed experimentally with different types of image datasets which are Oliva and Torralba dataset (OT8), Event sport datasets, 2D HeLa medical images, and also the new proposed scene data sets, namely, USM scene dataset. The experimental results showed the superiority of the proposed CLTP against the LBP, and CLBP. This is because the proposed CLTP is insensitive to noise as investigated and proved in the first experiment and has a high discriminating property that leads to achieve impressive classification accuracy rates.

\section{ACKNOWLEGMENT}

This work is supported by the Universiti Malaysia Pahang (UMP) via Research Grant UMP RDU160349. 


\section{REFERENCES}

[1] S. Murala, R. P. Maheshwari, and R. Balasubramanian, "Local tetra patterns: A new feature descriptor for content-based image retrieval," IEEE Transactions on Image Processing, vol. 21, no. 5, pp. 2874-2886, May 2012.

[2] J. Xiao, J. Hays, K. Ehinger, A. Oliva, and A. Torralba, "SUN database: Large-scale scene recognition from abbey to zoo," in Proceedings of the 2010 IEEE Computer Society Conference on Computer Vision and Pattern Recognition, 2010, pp. 3485 -3492.

[3] T. H. Rassem, N. M. Makbol, and S. Y. Yee, "Face recognition using completed local ternary pattern (CLTP) texture descriptor," International Journal of Electrical and Computer Engineering (IJECE), vol. 7, no. 3, 2017, pp. 1594-1601.

[4] X. Wang, T. Han, and S. Yan, "An HOG-LBP human detector with partial occlusion handling," in Proceedings of 12th IEEE International Conference of Computer Vision, 2009, pp. 32-39.

[5] J. Zhang and T. Tan, "Brief review of invariant texture analysis methods," Pattern Recognition, vol. 35, no. 3, pp. $735-747,2002$.

[6] T. Reed and J. Dubuf, "A review of recent texture segmentation and feature extraction techniques," CVGIP: Image Understanding, vol. 57, no. 3, pp. 359 - 372, 1993.

[7] R. W. Conners and C. A. Harlow, "A theoretical comparison of texture algorithms," IEEE Transactions on Pattern Analysis and Machine Intelligence, vol. PAMI-2, no. 3, pp. 204-222, May 1980.

[8] T. Ojala, M. Pietikinen, and D. Harwood, "A comparative study of texture measures with classification based on featured distributions," Pattern Recognition, vol. 29, no. 1, pp. 51 - 59, 1996.

[9] T. Ojala, M. Pietikainen, and T. Maenpaa, "Multiresolution gray-scale and rotation invariant texture classification with local binary patterns," IEEE Transactions on Pattern Analysis and Machine Intelligence, vol. 24, no. 7, pp. 971-987, July 2002.

[10] M. Mansourvar, M. A. Ismail, R. G. Raj, S. A. Kareem, S. Aik, R. Gunalan, C. D. Antony, The applicability of Greulich and Pyle atlas to assess skeletal age for four ethnic groups, In Journal of Forensic and Legal Medicine, Volume 22, 2014, Pages 26-29, ISSN 1752-928X, https://doi.org/10.1016/j.jflm.2013.11.011..

[11] S. Liao, M. Law, and A. Chung, "Dominant local binary patterns for texture classification," IEEE Transactions on Image Processing, vol. 18, no. 5, pp. 1107-1118, May 2009.

[12] X. Tan and B. Triggs, "Enhanced local texture feature sets for face recognition under difficult lighting conditions," IEEE Transactions on Pattern Analysis and Machine Intelligence, vol. 19, no. 6, pp. 16351650, June 2010.

[13] Z. Guo, L. Zhang, and D. Zhang, "A completed modeling of local binary pattern operator for texture classification," IEEE Transactions on Image Processing, vol. 19, no. 6, pp. 1657-1663, Jun 2010.

[14] F. Khalilah, "Texture classification using dominant neighborhood structure," IEEE Transactions on Image Processing, vol. 20, no. 11, pp. 3270-3279, Nov 2011.

[15] Y. Zhao, D.-S. Huang, and W. Jia, "Completed local binary count for rotation invariant texture classification,” IEEE Transactions on Image Processing, vol. 21, no. 10, pp. 4492-4497, Oct. 2012.

[16] T. H. Rassem and B. E. Khoo, "Completed local ternary pattern for rotation invariant texture classification,” The Scientific World Journal, vol. 2014, 2014. 
[17] A. Oliva and A. Torralba, "Modeling the shape of the scene: A holistic representation of the spatial envelope," International Journal of Computer Vision, vol. 42, no. 3, pp. 145-175, May 2001.

[18] L.-J. Li and L. Fei-Fei, "What, where and who classifying event by scene and object recognition?" in Proceedings of 11th International Conference in Computer Vision, 2007, pp. 1-8.

[19] M. V. Boland and R. F. Murphy, "A neural network classifier capable of recognizing the patterns of all major subcellular structures in fluorescence microscope images of Hela cells." Bioinformatics, vol. 17, no. 12, pp. 1213-1223, 2001.

[20] L. Nanni and S. Lumini, A. and Brahnam, "Local binary patterns variants as texture descriptors for medical image analysis," Artificial Intelligence in Medicine, vol. 49, no. 2, pp. 117-125, 2010.

[21] L. Nanni, S. Brahnam, and A. Lumini, "Selecting the best performing rotation invariant patterns in local binary/ternary patterns," in Proceed ings of 14th International Conference on Image Processing, Computer Vision, and Pattern Recognition, 2010, pp. 369-375.

[22] A. Chebira, Y. Barbotin, C. Jackson, T. Merryman, G. Srinivasa, R. F. Murphy, and J. Kovacevic, "A multiresolution approach to automated classification of protein subcellular location images," $B M C$ Bioinformaics, vol. 8, p. 210, 2007.

[23] Timo, O., T., M., M., P., J., V. \& S., K. J. . H. “Outex - new framework for empirical evaluation of texture analysis algorithms", in 'Proceedings of 16 th International Conference on Pattern Recognition', pp. 701-706, 2002.

[24] Qi X, Zhao G, Shen L, Li Q, Pietikainen M. LOAD: Local Orientation Adaptive Descriptor for Texture and Material Classification. arXiv preprint arXiv:1504.05809. 2015. 